\title{
Differential Equations
}

An Introduction to Basic Concepts, Results and Applications 


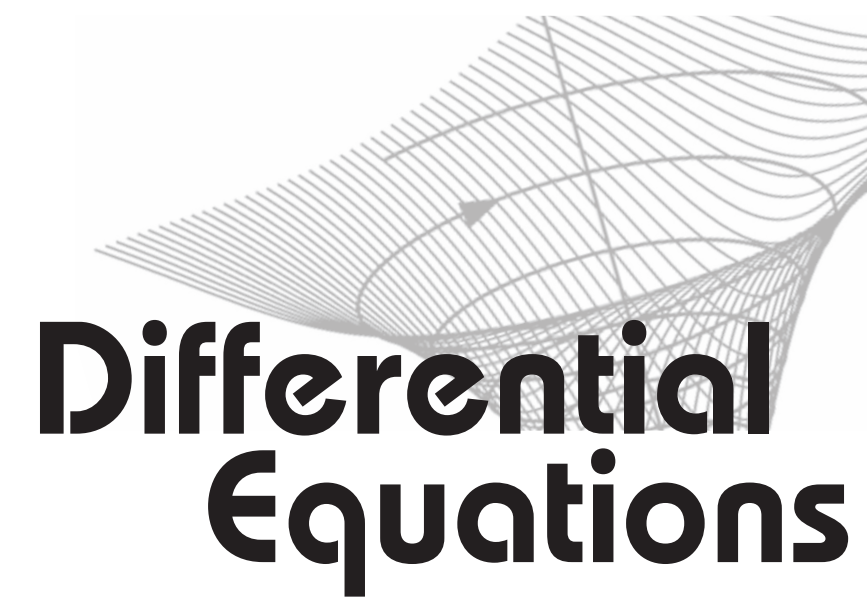

An Introduction to Basic Concepts, Results and Applications

\author{
Second Edition
}

\author{
loan I Vrabie \\ "Al. I. Cuza" University of Iasi and
"O. Mayer" Mathematics Institute of the
Romania Academy, Iasi, Romania \\ "Al. I. Cuza" University of Iasi and
"O. Mayer" Mathematics Institute of the
Romania Academy, Iasi, Romania \\ "Al. I. Cuza" University of Iasi and
"O. Mayer" Mathematics Institute of the
Romania Academy, Iasi, Romania
}


This page is intentionally left blank 


\section{Published by}

World Scientific Publishing Co. Pte. Ltd.

5 Toh Tuck Link, Singapore 596224

USA office: 27 Warren Street, Suite 401-402, Hackensack, NJ 07601

UK office: 57 Shelton Street, Covent Garden, London WC2H 9HE

\section{Library of Congress Cataloging-in-Publication Data}

Vrabie, I. I. (Ioan I.), 1951-

Differential equations : an introduction to basic concepts, results, and applications / by Ioan I

Vrabie. -- 2nd ed.

p. cm.

"Entirely rewritten English version of the lecture notes of a course on Differential Equations ... written in 1999 in Romanian"--Pref., 1st ed.

Includes bibliographical references and index.

ISBN-13: 978-981-4335-62-1 (hard cover : alk. paper)

ISBN-10: 981-4335-62-2 (hard cover : alk. paper)

1. Differential equations--Textbooks. I. Title.

QA371.V73132011

515 '.35--dc22

2011007636

\section{British Library Cataloguing-in-Publication Data}

A catalogue record for this book is available from the British Library.

Copyright (@ 2011 by World Scientific Publishing Co. Pte. Ltd.

All rights reserved. This book, or parts thereof, may not be reproduced in any form or by any means, electronic or mechanical, including photocopying, recording or any information storage and retrieval system now known or to be invented, without written permission from the Publisher.

For photocopying of material in this volume, please pay a copying fee through the Copyright Clearance Center, Inc., 222 Rosewood Drive, Danvers, MA 01923, USA. In this case permission to photocopy is not required from the publisher.

Printed in Singapore. 
To the Memory of my Parents 
This page is intentionally left blank 


\section{Preface to the Second Edition}

The Second Edition of the book differs from the First Edition in that several additions and changes were done. First, in order to offer the reader some basic information concerning two important topics which, in one way or another, are related to differential equations, the following insertions were operated. So, keeping in mind that Volterra Equations are not only nontrivial extensions of differential equations but one of the starting points of research in Operator Theory, we have included Chapter 8, entirely devoted to this old but still modern and of great interest topic. Once this being done, taking into consideration that a very efficient tool in solving linear integral equations of Volterra type is the Laplace Transform, we have decided to present the fundamentals of this theory in Section 10.4. Further, we have added Chapter 9 dealing with the Calculus of Variations, a discipline having its roots in Mechanics and which is essentially based on nonlinear differential equations. It is almost needless to say that the Calculus of Variations is indispensable not only for mathematicians but for physicists as well. In addition, this branch of Mathematics was - and still is - the starting point of many deep researches in both Differential Equations and Differential Geometry.

As in the case of all other chapters, each of Chapters 8 and 9 ends with an exercises and problems section. The complete solutions of the exercises and problems newly added were included in the last section of the book.

Second, trying to make the book as self-contained as possible, a simple form of the Implicit Functions Theorem, i.e. Theorem 2.8.1, whose proof was derived from Peano's Theorem 2.2.1, was included. In addition, an extension to the case of implicitly defined vector-valued functions was proposed in Problem 2.28. It should be noticed that the proof here presented, in spite of its relative simplicity - due, of course, to the very deep result 
on which is based, i.e. Peano's Theorem 2.2.1 -, is, with no doubt, far from being elementary. However, the reader which is familiar with Peano's Theorem 2.2.1 would have no problem in following the arguments there involved.

It should be emphasized that both newly added chapters and sections are intended as an incentive for further study rather than a main source of information.

Several new entries on the reference list were included. These are [Arnold (1978)], [Barbu (2010)], [Bliss (1946)], [Cârjă et al. (2007)], [Gelfand and Fomin (1963)], [Giusti (2003)], [Havârneanu (2007)], [Krasnov et al. (1977)], [Lavrentiev and Sabat (1959)], [Lefter (2006a)], [Lefter (2006b)], [Sneddon (1971)] [Şabac (1981)], [Widder (1946)] and two of them, i.e. [Cârjă et al. (2004)] and [Cârjă and Vrabie (2005)], which were just in print at the time when the First Edition has appeared, were updated. Some small corrections were operated and a direct proof of the implication (iii) implies (i) in Theorem 4.1.4 was included, substituting the indirect proof given in the First Edition.

Acknowledgements. I gratefully acknowledge the help of Professors Teodor Havârneanu, Cătălin-George Lefter and Mihai Necula, from the Department of Mathematics of "Al. I. Cuza" University of Iaşi, and that of Professor Marius Popescu, from the Department of Mathematics of the University of Galaţi, for the very careful reading of the newly added parts to the Second Edition of the book, and for the useful suggestions and remarks they have made. All their comments, which I took into consideration, have led to a substantial improvement of the presentation. I would also like to mention that Example 9.4.1 is due to Professor Eugen Popa, from the Department of Mathematics of "Al. I. Cuza" University of Iaşi.

I take this opportunity to express my warmest thanks to all of them as well as to the editors of World Scientific for the excellent job of proofreading the whole manuscript. 


\section{Preface to the First Edition}

The book is an entirely rewritten English version of the lecture notes of a course on Differential Equations I taught during the last twelve years at the Faculty of Mathematics of "Al. I. Cuza" University of Iaşi. These lecture notes were written in 1999 in Romanian. Their goal was to present in a unitary frame and from a new perspective the main concepts and results belonging to a discipline which, due to the continuous interplay between theory and applications, is by far one of the most fascinating branches of modern mathematics, i.e. differential equations. It was my intention to give the reader the opportunity to know a point of view — rather different from the traditional one - offering a possible way to learn differential equations with main emphasis on the Cauchy problem. So, I decided to treat separately the problems of: existence, uniqueness, approximation, continuation of the solutions and, at the same time, to give the simplest possible but complete proofs to some fundamental results which are at the core of the discipline: Peano's local existence theorem, the classification of non-continuable solutions from the viewpoint of their behavior at the end of the existence interval, the continuous dependence of the solution on the data and parameters, etc. This goal was by far very hard to accomplish due to the existence of a long list of very good, or even exceptional, textbooks and monographs on this subject covering all levels of difficulty: [Arnold (1974)], [Arrowsmith and Place (1982)], [Barbu (1985)], [Braun (1983)], [Coddington and Levinson (1955)], [Corduneanu (1977)], [Cronin (1980)], [Elsgolts (1980)], [Halanay (1972)], [Hale (1969)], [Hartman (1964)], [Hirsch and Smale (1974)], [Hubbard and West (1995)], [Ionescu (1972)], [Piccinini et al.. (1984)], [Pontriaghin (1969)], to cite only a few. However, in spite of this challenging competition, I hope that the reader will find this text attractive enough from both the viewpoint of the chosen topics and 
the presentation.

The book contains a preface, a list of symbols, seven main chapters, a short chapter on auxiliary results, a rather long section including detailed solutions to all exercises and problems, a bibliography and ends with an index. With the sole exception of Chapters 6 and 7 , which require some basic results on Lebesgue integral and Measure Theory, it is completely accessible to any reader having satisfactory knowledge of Linear Algebra and Mathematical Analysis. The 36 figures included illustrate the concepts introduced and smooth the way towards a complete understanding of the arguments used in the proofs.

The first chapter starts with a very brief presentation of the main steps made along the last four centuries toward the modern theory of differential equations. It continues with some preliminary notions and results referring to: the concept of solution, some methods of solving elementary equations, various mathematical models described either by differential equations or systems of differential equations, and some basic integral inequalities.

The second chapter contains several fundamental results concerning the Cauchy Problem: the local existence, the continuation of the solutions, the existence of global solutions, the relationship between the local and the global uniqueness, the continuous dependence and the differentiability of the solutions with respect to the data and to the parameters.

The third chapter is merely concerned with some classical facts about the approximation of the solutions: the method of power series, the method of successive approximations, the method of polygonal lines, the implicit Euler method and a particular, and therefore simplified, instance of Crandall-Liggett exponential formula.

In the fourth chapter we apply the previously developed theory to a systematic study of one of the most important class of systems: first-order linear differential systems. Here we present the main results concerning the global existence and uniqueness, the structure of the space of solutions, the fundamental matrix and the Wronskian, the variation of constants formula, the properties of the mapping $t \mapsto e^{t \mathcal{A}}$ and the basic results referring to $n^{\text {th }}$-order linear differential equations.

The fifth chapter is mainly concerned with the study of an extremely important problem of the discipline: the stability of solutions. We introduce four concepts of stability and we successively study the stability of the null solution of linear systems, perturbed systems and fully nonlinear systems respectively, in the last case by means of the Lyapunov's function method. We also include some facts about instability which is responsible for the 
so-called unpredictability and chaos.

In the sixth chapter, we start with the study of the concept of prime integral, first for autonomous, and thereafter for non-autonomous systems. Next, with this background material at hand, we present the basic results concerning linear and quasi-linear first-order partial differential equations. Some examples of conservation laws are also included.

The seventh chapter, rather heterogeneous, has a very special character being conceived to help the reader to go deeper within this discipline. So, here, we discuss some concepts and results concerning distributions and solutions in the sense of distributions, Carathéodory solutions, differential inclusions, variational inequalities, viability and invariance and gradient systems.

In the last chapter we include some auxiliary concepts and results needed for a good understanding of some parts of the book: the operator norm of a matrix, compact sets in $C\left([a, b] ; \mathbb{R}^{n}\right)$, the projection of a point on a convex set.

Each chapter, except the one on Auxiliary Results, ends with a special section containing exercises and problems ranging from extremely simple to challenging ones. The complete proofs of all these are included into a rather developed final section (more than 60 pages).

Acknowledgements. The writing of this book was facilitated by a very careful reading of some parts of the manuscript, by several remarks and suggestions made by Professors Ovidiu Cârjă, Mihai Necula from "Al. I. Cuza" University of Iaşi, by Professors Silvia-Otilia Corduneanu and Silviu Nistor from "Gh. Asachi" Technical University of Iaşi, remarks and suggestions which I took into account. The simplified version of the Frobenius theorem was called to my attention by Dr. Constantin Vârsan, Senior Researcher at The Mathematical Institute of the Romanian Academy in Bucharest. Some of the examples in Physics and Chemistry have been reformulated taking into account the remarks made by Professors Dumitru Luca and Gelu Bourceanu. Professor Constantin Onică had a substantial contribution in solving and correcting most part of the exercises and the problems proposed. A special mention deserves the very careful — and thus critical — reading of the English version by Professor Mircea Bârsan. It is a great pleasure to express my appreciation to all of them. 
This page is intentionally left blank 


\title{
List of Symbols
}

\author{
$\mathcal{A}^{\tau}$ \\ $B(\xi, r)$ \\ $\stackrel{\circ}{B}$ \\ $\mathcal{B}^{*}$ \\ $\operatorname{conv} F$ \\ $\overline{\operatorname{conv} F}$ \\ - the transpose of the matrix $\mathcal{A} \in \mathcal{M}_{n \times n}(\mathbb{R})$ \\ $\mathcal{e P}(\mathbb{I}, \Omega, f, a, \xi)-$ \\ the closed ball centered at $\xi$ of radius $r>0$ \\ - the interior of the set $B$ \\ - the adjoint of the matrix $\mathcal{B} \in \mathcal{M}_{n \times n}(\mathbb{R})$ \\ $C\left([a, b] ; \mathbb{R}^{n}\right) \quad-$ \\ the convex hull of $F$, i.e. the set of all convex \\ combinations of elements in $F$ \\ $\mathcal{D}$ \\ $\operatorname{dist}(\mathcal{K}, \mathcal{F})$ \\ $\operatorname{dist}(\eta, \mathcal{K})$ \\ the closed convex hull of $F$, i.e. the closure of $\operatorname{conv} F$ \\ the Cauchy problem $x^{\prime}=f(t, x), x(a)=\xi$, where \\ $f: \mathbb{I} \times \Omega \rightarrow \mathbb{R}^{n}, a \in \mathbb{I}$ and $\xi \in \Omega$ \\ the space of continuous functions from $[a, b]$ to $\mathbb{R}^{n}$ \\ the data $(\mathbb{I}, \Omega, f, a, \xi)$. So, $\mathcal{C P}(\mathcal{D})$ denotes $\mathcal{C P}(\mathbb{I}, \Omega, f, a, \xi)$ \\ the distance between the sets $\mathcal{K}$ and $\mathcal{F}$, i.e. \\ $\operatorname{dist}(\mathcal{K}, \mathcal{F})=\inf \{\|x-y\| ; x \in \mathcal{K}, y \in \mathcal{F}\}$ \\ $\mathcal{D}(\mathbb{R}) \quad$ - $\quad$ the space of indefinite differe \\ the distance between the point $\eta$ and the set $\mathcal{K}$, i.e. \\ $\operatorname{dist}(\eta, \mathcal{K})=\inf \{\|\eta-\xi\| ; \xi \in \mathcal{K}\}$ \\ $\Delta$ \\ $\delta J[q][\eta]$ \\ - the compact set $[a, a+h] \times B(\xi, r)$ \\ $\delta^{2} J[q][\eta]$ \\ $\mathcal{D}^{\prime}(\mathbb{R})$ \\ $\operatorname{graph}(x)$ \\ $\mathbb{I}, \mathbb{J}, \mathbb{K}$ \\ $\mathcal{L}[f(t)](p)$ \\ $\mathbb{N}$ \\ $\mathbb{N}^{*} \quad-\quad$ the set of positive integers, i.e. $1,2,3, \ldots$ \\ - the first variation of $J$ calculated at $q$ along the direction $\eta$, \\ i.e. $\left.\frac{d}{d \varepsilon} J[q+\varepsilon \eta]\right|_{\varepsilon=0}$ \\ - the second variation of $J$ calculated at $q$ along the direction \\ $\eta$, i.e. $\left.\frac{d^{2}}{d \varepsilon^{2}} J[q+\varepsilon \eta]\right|_{\varepsilon=0}$ \\ - the set of linear continuous functionals from $\mathcal{D}(\mathbb{R})$ to $\mathbb{R}$ \\ — the graph of $x: \mathbb{I} \rightarrow \mathbb{R}^{n}$, i.e. graph $(x)=\{(t, x(t)) ; t \in \mathbb{I}\}$ \\ - nonempty intervals in $\mathbb{R}$ \\ - the Laplace Transform of the function $f$ \\ $\nabla_{x} z$, or $\nabla z \quad$ - the gradient of the function $z$ with respect to $x_{1}, x_{2}, \ldots, x_{n}$, \\ $\|\mathcal{A}\|_{\mathcal{M}}$ \\ i.e. $\nabla_{x} z=\operatorname{grad}_{x} z=\left(\frac{\partial z}{\partial x_{1}}, \frac{\partial z}{\partial x_{2}}, \ldots, \frac{\partial z}{\partial x_{n}}\right)$ \\ - the norm of the matrix $\mathcal{A} \in \mathcal{M}_{n \times m}(\mathbb{R})$, i.e. \\ $\|\mathcal{A}\|_{\mathcal{M}}=\sup \left\{\|\mathcal{A} x\|_{n} ; x \in \mathbb{R}^{m},\|x\|_{m} \leq 1\right\}$
}


$\Omega$

$\omega_{f}$

$\mathcal{P}_{K}(x)$

$\mathbb{R}$

$\mathbb{R}^{*}$

$\mathbb{R}_{+}$

$\mathbb{R}_{+}^{*}$

$\operatorname{supp} \phi$

$\Sigma$

$x^{\prime}$

$\dot{x}$

$x^{-1}(s)$
- a nonempty and open subset in $\mathbb{R}^{n}$

- the growth index of the function $f$, i.e. $\omega_{f}=\inf \left\{\omega \in \mathbb{R} ; \exists M>0,|f(t)| \leq M e^{\omega t}, \forall t>0\right\}$

- the projection of the vector $x \in \mathbb{R}^{n}$ on the subset $K \subset \mathbb{R}^{n}$

- the set of real numbers

- the set of real numbers excluding 0

- the set of nonnegative real numbers

- the set of positive real numbers

- the set $\overline{\{t \in \mathbb{R} ; \phi(t) \neq 0\}}$

- a nonempty and locally closed subset in $\Omega$

- the derivative of the function $x$

- the derivative in the sense of distributions of the distribution $x$

- the inverse of the matrix $X(s)$, i.e. $X^{-1}(s)=[X(s)]^{-1}$ 


\section{Contents}

Preface to the Second Edition vii

Preface to the First Edition ix

List of Symbols xiii

1. Generalities 1

1.1 Brief History . . . . . . . . . . . . . . . . . 1

1.1.1 The Birth of the Discipline . . . . . . . . . 1

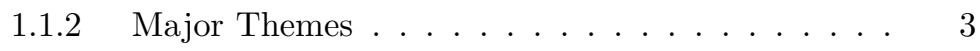

1.2 Introduction . . . . . . . . . . . . . . . . . 11

1.3 Elementary Equations . . . . . . . . . . . . . 18

1.3.1 Equations with Separable Variables . . . . . . . . 19

1.3.2 Linear Equations . . . . . . . . . . . . . . 20

1.3.3 Homogeneous Equations . . . . . . . . . . 21

1.3.4 Bernoulli Equations . . . . . . . . . . . . 22

1.3.5 Riccati Equations . . . . . . . . . . . . 23

1.3.6 Exact Differential Equations . . . . . . . . . . 24

1.3.7 Equations Reducible to Exact Differential Equations 25

1.3.8 Lagrange Equations . . . . . . . . . . . . . 25

1.3.9 Clairaut Equations . . . . . . . . . . . . 26

1.3.10 Higher-Order Differential Equations . . . . . . . . 27

1.4 Some Mathematical Models . . . . . . . . . . . . . . 29

1.4.1 Radioactive Disintegration . . . . . . . . . . 30

1.4.2 The Carbon Dating Method . . . . . . . . . . 30

1.4.3 Equations of Motion . . . . . . . . . . 31

1.4.4 The Harmonic Oscillator . . . . . . . . . . . . 31 
1.4.5 The Mathematical Pendulum . . . . . . . . . . 32

1.4.6 Two Demographic Models . . . . . . . . . . . 33

1.4.7 A Spatial Model in Ecology . . . . . . . . . . . 35

1.4.8 The Prey-Predator Model . . . . . . . . . . . . . 35

1.4.9 The Spreading of a Disease . . . . . . . . . . . 37

1.4 .10 Lotka Model . . . . . . . . . . . . . . . . . . . 40

1.4.11 An Autocatalytic Generation Model . . . . . . . . 41

1.4.12 An RLC Circuit Model . . . . . . . . . . . . . 41

1.5 Integral Inequalities . . . . . . . . . . . . . . . . 43

$1.6 \quad$ Exercises and Problems . . . . . . . . . . . . . . 45

2. The Cauchy Problem 49

2.1 General Presentation . . . . . . . . . . . . . . . . . . . . 49

2.2 The Local Existence Problem . . . . . . . . . . . . . . . 55

2.3 The Uniqueness Problem . . . . . . . . . . . . . . . 59

2.3.1 The Locally Lipschitz Case . . . . . . . . . . . . 60

2.3 .2 The Dissipative Case . . . . . . . . . . . . . . 62

2.4 Saturated Solutions. . . . . . . . . . . . . . . 63

2.4.1 Characterization of Continuable Solutions . . . . 64

2.4.2 The Existence of Saturated Solutions . . . . . . . 65

2.4.3 Types of Saturated Solutions . . . . . . . . . . 66

2.4.4 The Existence of Global Solutions . . . . . . . . 70

2.5 Continuous Dependence on Data and Parameters . . . . . 73

2.5.1 The Dissipative Case . . . . . . . . . . . . 75

2.5.2 The Locally Lipschitz Case . . . . . . . . . . . . 77

2.5.3 Continuous Dependence on Parameters . . . . . . 79

2.6 Problems of Differentiability . . . . . . . . . . . . . 81

2.6.1 Differentiability with Respect to the Data . . . . 82

2.6.2 Differentiability with Respect to the Parameters . 85

2.7 The Cauchy Problem for the $n^{\text {th }}$-Order Differential Equation 89

2.8 The Implicit Function Theorem . . . . . . . . . . . . . . . 91

2.9 Exercises and Problems _... . . . . . . . . . . 93

3. Approximation Methods 99

3.1 The Power Series Method . . . . . . . . . . . . . . . . . 99

$3.1 .1 \quad$ An Example . . . . . . . . . . . . . . . . . 99

3.1.2 The Existence of Analytic Solutions . . . . . . . 100

3.2 The Successive Approximations Method . . . . . . . . . 105 
3.3 The Method of Polygonal Lines . . . . . . . . . . . . . . 108

3.4 The Euler Implicit Method. The Exponential Formula . . 112

3.4.1 The Semigroup Generated by $\mathcal{A}$. . . . . . . . 112

3.4.2 Two Auxiliary Lemmas . . . . . . . . . . . . . . . 114

3.4.3 The Exponential Formula . . . . . . . . . . . . . 116

$3.5 \quad$ Exercises and Problems . . . . . . . . . . . . . 120

4. Systems of Linear Differential Equations 123

4.1 Homogeneous Systems. The Space of Solutions . . . . . . 123

4.2 Non-homogeneous Systems. The Variation of Constants

Formula . . . . . . . . . . . . . . . . . 132

4.3 The Exponential of a Matrix . . . . . . . . . . . . 134

4.4 A Method to Find $e^{t \mathcal{A}} \ldots \ldots \ldots \ldots . \ldots \ldots$

4.5 The $n^{\text {th }}$-Order Linear Differential Equation $\ldots \ldots \ldots . . .142$

4.6 The $n^{\text {th }}$-Order Linear Differential Equation with Constants Coefficients . . . . . . . . . . . . . 147

4.7 Exercises and Problems . . . . . . . . . . . . . . 151

5. Elements of Stability 155

5.1 Types of Stability . . . . . . . . . . . . . . . . 155

5.2 Stability of Linear Systems _. . . . . . . . . . . . 161

5.3 The Case of Perturbed Systems . . . . . . . . . . . . . . 169

5.4 The Lyapunov Function Method . . . . . . . . . . . . . 173

5.5 The Case of Dissipative Systems . . . . . . . . . . . . . 181

5.6 The Case of Controlled Systems . . . . . . . . . . . . . . 187

5.7 Unpredictability and Chaos . . . . . . . . . . . . . . 190

5.8 Exercises and Problems . . . . . . . . . . . . . . 197

6. Prime Integrals 201

6.1 Prime Integrals for Autonomous Systems _ . . . . . . . . 201

6.2 Prime Integrals for Non-Autonomous Systems . . . . . . . 211

6.3 First Order Partial Differential Equations . . . . . . . . . 212

6.4 The Cauchy Problem for Quasi-Linear Equations . . . . . 216

6.5 Conservation Laws . . . . . . . . . . . . . . . . . . 221

6.5.1 Some Examples . . . . . . . . . . . . . . 221

6.5.2 A Local Existence and Uniqueness Result . . . . . 223

6.5.3 Weak Solutions . . . . . . . . . . . . . . . . 224

6.6 Exercises and Problems . . . . . . . . . . . . . 233 
7. Extensions and Generalizations 239

$7.1 \quad$ Distributions of One Variable . . . . . . . . . . . . . 239

7.2 The Convolution Product . . . . . . . . . . . . . . . 248

7.3 Generalized Solutions . . . . . . . . . . . . . . 251

7.4 Carathéodory Solutions . . . . . . . . . . . . . . 258

7.5 Differential Inclusions . . . . . . . . . . . . . . . . . . . . 264

7.6 Variational Inequalities . . . . . . . . . . . . . . . 272

7.7 Problems of Viability . . . . . . . . . . . . . . . . . . 279

7.8 Proof of the Nagumo's Viability Theorem . . . . . . . . . 281

7.9 Sufficient Conditions for Invariance . . . . . . . . . . . . . 287

7.10 Necessary Conditions for Invariance . . . . . . . . . . . . 290

7.11 Gradient Systems. Frobenius Theorem . . . . . . . . . . . 294

7.12 Exercises and Problems . . . . . . . . . . . . 300

8. Volterra Equations 305

8.1 Volterra Equations of the Second Kind . . . . . . . . . . . 305

8.2 The Resolvent Kernel . . . . . . . . . . . . . . . . . . . . 308

8.3 Volterra Equations of the First Kind . . . . . . . . . . . . 310

8.4 The Nonlinear Case . . . . . . . . . . . . . . . . . . . . . 311

8.5 Exercises and Problems . . . . . . . . . . . . . 314

9. Calculus of Variations 319

9.1 Some Examples . . . . . . . . . . . . . . . . . . . . . 319

9.2 Necessary Conditions for Extremum . . . . . . . . . . . . 323

9.2.1 The Scalar-Valued Case . . . . . . . . . . . 325

9.2.2 The Vector-Valued Case . . . . . . . . . . . . . 328

9.3 Some Particular Cases . . . . . . . . . . . . . . . . . 330

9.4 Regularity of the Extremals . . . . . . . . . . . . . . 331

9.5 Higher-Order Necessary Conditions . . . . . . . . . . . . . 335

9.6 Sufficient Conditions for Extremum . . . . . . . . . . . . . 341

9.7 The Canonical Euler-Lagrange System . . . . . . . . . . . 343

9.8 Prime Integrals of the Euler-Lagrange System . . . . . . . 345

9.9 Exercises and Problems . . . . . . . . . . . . 346

10. Auxiliary Results 349

10.1 Elements of Vector Analysis . . . . . . . . . . . . . . . . . 349

10.2 Compactness in $C\left([a, b] ; \mathbb{R}^{n}\right)$. . . . . . . . . . . 355

10.3 The Projection of a Point on a Convex Set . . . . . . . . 360 
10.4 The Laplace Transform . . . . . . . . . . . . . . . . 362 10.4.1 The Transforms of Some Elementary Functions 370

Solutions 373

Chapter 1. . . . . . . . . . . . . . . . . . . 373

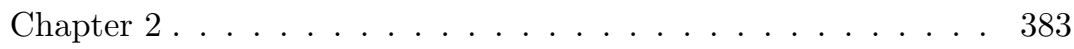

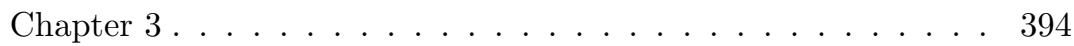

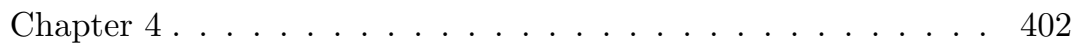

Chapter 5 . . . . . . . . . . . . . . . . . . 4 410

Chapter 6. . . . . . . . . . . . . . . . . . . . . 4 419

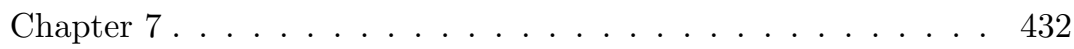

Chapter 8. . . . . . . . . . . . . . . . 437

Chapter $9 \ldots \ldots \ldots \ldots \ldots \ldots$. . . . . . . . . . . . . . . . . . . . .

$\begin{array}{ll}\text { Bibliography } & 451\end{array}$

$\begin{array}{lll}\text { Index } & 455\end{array}$ 